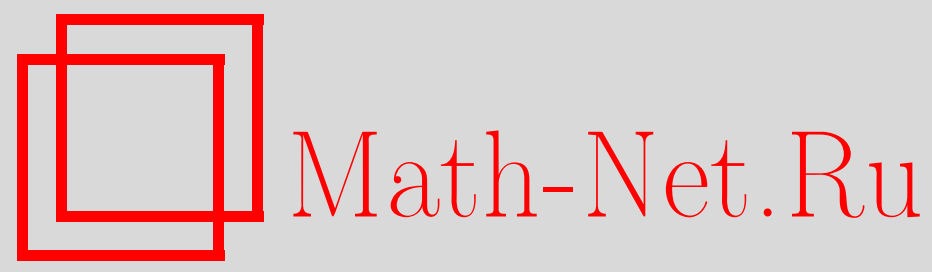

А. А. Рябинин, В. Д. Быстрицкий, В. А. Ильичев, О сингулярных строго монотонных функциях, Матем. заметки, 2004, том 76, выпуск 3, 439-451

DOI: https://doi.org/10.4213/mzm120

Использование Общероссийского математического портала Math-Net.Ru подразумевает, что вы прочитали и согласны с пользовательским соглашением http://www . mathnet.ru/rus/agreement

Параметры загрузки:

IP: 54.147 .182 .235

26 апреля 2023 г., 15:40:41

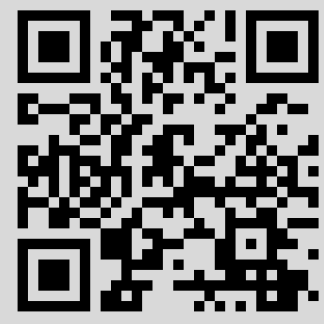




\title{
о СИНГУЛЯРНЫХ СТРОГО МОНОТОННЫХ ФУНКЦИЯХ
}

\author{
А.А. Рябинин, В. Д. Быстрицкий, В. А. Ильичев
}

\begin{abstract}
Описывается универсальная методика построения непрерьвных строго монотонно возрастающих сингулярных функций на отрезке $[-1,1]$. "Генератором" метода является ряд $\sum_{k=1}^{\infty} \pm 2^{-k}$ со случайной расстановкой знаков, и соответствующие функции порождаются как функции распределения таких рядов. В качестве примеров рассматриваются два стохастических способа расстановки знаков: независимый и марковский.

Библиография: 10 названий.
\end{abstract}

1. Введение. Непрерывная функция $F(x)$, определенная на отрезке $[-1,1]$ и имеющая ограниченную вариацию, называется сингулярной, если $F^{\prime}(x)=0$ почти всюду на $[-1,1]$. Нас будут интересовать сингулярные монотонные функции, удовлетворяюшие условиям $F(-1)=0, F(1)=1$. Продолжим $F(x)$ за пределы $[-1,1]$ по правилу: $F(x)=0$ при $x<-1$ и $F(x)=1$ при $x>1$. Точка $x \in[-1,1]$ называется точкой роста $F(x)$, если для произвольного $\epsilon>0 F(x+\epsilon)-F(x-\epsilon)>0$. Совокупность точек роста функции $F(x)$ называется спектром функции и обозначается $\operatorname{Sp}(F)$. Спектр непрерывной функции является совершенным множеством.

Как следует из названия статьи, нас интересуют строго монотонно возрастаюшие сингулярные функции, т.е. такие функции, спектром которых является весь отрезок $[-1,1]$. Существование таких функций весьма не очевидно, в отличие от сингулярных функций типа "канторовской лестницы", для которых спектр представляет из себя совершенное нигде не плотное множество лебеговой меры нуль. Одним из первых примеров сингулярной строго монотонной функции явилась функция Минковского [1] (см. также [2]), достаточно полное исследование свойств которой, включая и доказательство сингулярности, было проведено А. Данжуа [3]. Другой пример, на котором мы хотим заострить внимание, был построен Р. Салемом [2]. В [4, с. 59] приведена несколько видоизмененная процедура построения функции Салема (так впредь мы будем ее называть). В ином контексте функция Салема появляется в работе [5] в качестве решения одного функционального уравнения. В книге [6, с. 155$]$ приведена процедура построения сингулярных строго монотонных функций, основу которой составляют сингулярные функции типа "канторовской лестницы" и метод "сгущения особенностей".

В данной статье описывается вероятностная процедура построения всего класса сингулярных строго монотонных функций. Ключевым понятием в этой модели являются ряды со случайной расстановкой знаков

$$
\sum_{k=1}^{\infty} \pm \frac{1}{2^{k}}
$$

(C) А.А. Рявинин, В. Д. Быстрицкий, В.А. Ильичев 
и интересующие нас функции предстают как функции распределения сумм таких рядов. В частности, функция Салема порождается этой процедурой при независимом неравновероятном способе расстановки знаков.

2. Построение вероятностной процедуры. Пусть $\Omega=\left\{\omega: \omega=\left(t_{1}, t_{2}, \ldots\right.\right.$, $\left.\left.t_{n}, \ldots\right), t_{n}= \pm 1\right\}$. В построении процедуры можно выделить два существенных момента: во-первых, задание на $\Omega$ отображения $S: \Omega \rightarrow[-1,1]$ и, во-вторых, определение на $\Omega$ структуры вероятностного пространства $(\Omega, \beta, \mathrm{P})$, позволяющей рассматривать $S$ как случайную величину, функция распределения которой при определенных условиях будет строго монотонной сингулярной функцией.

Начнем с отображения $S$, которое определим формулой

$$
S(\omega)=\sum_{k=1}^{\infty} \frac{t_{k}}{2^{k}}, \quad \omega=\left(t_{1}, \ldots, t_{k}, \ldots\right)
$$

ЛЕмма 1. Образом $\Omega$ при отображсени $S$ будет отрезок $[-1,1]$, т.е. $S(\Omega)=$ $[-1,1]$.

ДоказАТЕЛЬСтво. Включение $S(\Omega) \subset[-1,1]$ очевидно. Покажем, что и $[-1,1] \subset$ $S(\Omega)$, т.е. для произвольной точки $x \in[-1,1]$ найдется $\omega_{x} \in \Omega$ такое, что

$$
S\left(\omega_{x}\right)=x \text {. }
$$

Заметим, что функция $u \rightarrow 1-2 u$ осуществляет биекцию отрезков $[0,1]$ и $[-1,1]$, поэтому, если

$$
u=\frac{\epsilon_{1}}{2}+\cdots+\frac{\epsilon_{k}}{2^{k}}+\cdots, \quad \epsilon_{k}=0 \text { или } 1,
$$

- двоичное разложение числа $u \in[0,1]$, то

$$
x=1-2 u=\frac{1-2 \epsilon_{1}}{2}+\cdots+\frac{1-2 \epsilon_{k}}{2^{k}}+\cdots=\frac{t_{1}^{(x)}}{2}+\cdots+\frac{t_{k}^{(x)}}{2^{k}}+\cdots,
$$

где $t_{k}^{(x)}=+1$ или -1 . Обозначая $\omega_{x}=\left(t_{1}^{(x)}, \ldots, t_{k}^{(x)}, \ldots\right)$, имеем $S\left(\omega_{x}\right)=x$. Лемма установлена.

ЗАмЕчАниЕ. Допуская, что сделано то или иное соглашение, обеспечивающее однозначность представления (3), можно утверждать, что посредством (1) устанавливается биекция между $\Omega$ и $[-1,1]$.

Особую роль для нас будут играть, так называемые, цилиндрические множества в $\Omega$.

ОПРЕДЕЛЕНИЕ. Подмножество из $\Omega$ вида

$$
C_{n}\left(\delta_{1}, \ldots, \delta_{n}\right)=\left\{\omega: \omega=\left(\delta_{1}, \ldots, \delta_{n} ; t_{n+1}, \ldots\right)\right\}
$$

где $\delta_{k}= \pm 1,1 \leqslant k \leqslant n$, фиксированы, а $t_{k}= \pm 1, k \geqslant n+1$, произвольные, называется иилиндрическим множеством (сокрашенно иилиндром) с основанием $\left(\delta_{1}, \ldots, \delta_{n}\right)$.

ЛЕмма 2. Образом иилиндра $C_{n}\left(\delta_{1}, \ldots, \delta_{n}\right)$ при отображсении $S$ является отрезок

$$
\left\{x \in[-1,1]:\left|x-x_{n}\right| \leqslant \frac{1}{2^{n}}, x_{n}=\frac{\delta_{1}}{2}+\frac{\delta_{2}}{2^{2}}+\cdots+\frac{\delta_{n}}{2^{n}}\right\} .
$$


ДокАЗАТЕЛЬСтво. Согласно лемме 1 и замечания к ней произвольному $\omega \in C_{n}\left(\delta_{1}\right.$, $\left.\ldots, \delta_{n}\right)$ взаимнооднозначно ставится в соответствие точка $x \in[-1,1]$ по правилу

$$
x=\frac{\delta_{1}}{2}+\cdots+\frac{\delta_{n}}{2^{n}}+\frac{t_{n+1}}{2^{n+1}}+\frac{t_{n+2}}{2^{n+2}}+\cdots=x_{n}+\frac{1}{2^{n}}\left(\frac{t_{n+1}}{2}+\frac{t_{n+2}}{2^{2}}+\cdots\right) .
$$

Здесь $t_{n+1}, t_{n+2}, \ldots$ принимают произвольные значения \pm 1 и в силу леммы 1 числа $\left(t_{n+1} / 2+t_{n+2} / 2^{2}+\cdots\right)$ сплош заполняют отрезок $[-1,1]$. Таким образом, соответствуюшие $x$ образуют отрезок $\left[x_{n}-1 / 2^{n}, x_{n}+1 / 2^{n}\right]$. Лемма установлена.

$\mathrm{C}$ помощью цилиндрических множеств определим на $\Omega$ структуру борелевского пространства $(\Omega, \beta)$, а именно, пусть $\beta-\sigma$-алгебра, порожденная цилиндрическими множествами $\left\{C_{n}\left(\delta_{1}, \ldots, \delta_{n}\right)\right\}$. Далее, на $(\Omega, \beta)$ определим вероятностную меру $\mathrm{P}$ посредством последовательности конечномерных вероятностньх распределений $\left\{P_{n}\left(t_{1}, \ldots, t_{n}\right)\right\}$, удовлетворяющих условию согласованности [7]: для $n=1,2, \ldots$

$$
P_{n}\left(t_{1}, \ldots, t_{n}\right)=P_{n+1}\left(t_{1}, \ldots, t_{n}, 1\right)+P_{n+1}\left(t_{1}, \ldots, t_{n},-1\right),
$$

так что

$$
\mathrm{P}\left(C_{n}\left(\delta_{1}, \ldots, \delta_{n}\right)\right)=P_{n}\left(\delta_{1}, \ldots, \delta_{n}\right), \quad n=1,2, \ldots
$$

Впредь подчиним последовательность $\left\{P_{n}\left(t_{1}, \ldots, t_{n}\right)\right\}$ условию

$$
\lim _{n \rightarrow \infty} P_{n}\left(t_{1}, \ldots, t_{n}\right)=0 \quad \forall \omega=\left(t_{1}, \ldots, t_{n}, \ldots\right) \in \Omega,
$$

которое означает, что вероятностное пространство $(\Omega, \beta, \mathrm{P})$ не имеет атомов. Действительно, для всех $\omega=\left(\delta_{1}, \ldots, \delta_{n}, \ldots\right) \in \Omega$

$$
\begin{aligned}
\mathrm{P}(\{\omega\}) & =\mathrm{P}\left(\bigcap_{n=1}^{\infty} C_{n}\left(\delta_{1}, \ldots, \delta_{n}\right)\right)=\lim _{n \rightarrow \infty} \mathrm{P}\left(C_{n}\left(\delta_{1}, \ldots, \delta_{n}\right)\right) \\
& =\lim _{n \rightarrow \infty} P_{n}\left(\delta_{1}, \ldots, \delta_{n}\right)=0 .
\end{aligned}
$$

Пусть $\left\{X_{k}(\omega)=t_{k}\right\}_{k=1}^{\infty}-$ последовательность координатных функций на $(\Omega, \beta, \mathrm{P})$. Для совместного распределения $\left(X_{1}, \ldots, X_{n}\right)$ имеем

$$
\begin{aligned}
\mathrm{P}\left(\omega: X_{1}=\delta_{1}, \ldots, X_{n}=\delta_{n}\right) & =\mathrm{P}\left(\omega: \omega=\left(\delta_{1}, \ldots, \delta_{n} ; t_{n+1}, \ldots\right)\right) \\
& =\mathrm{P}\left(C_{n}\left(\delta_{1}, \ldots, \delta_{n}\right)\right)=P_{n}\left(\delta_{1}, \ldots, \delta_{n}\right) .
\end{aligned}
$$

$\mathrm{Ha}(\Omega, \beta, \mathrm{P})$ рассмотрим ряд

$$
S(\omega)=\sum_{k=1}^{\infty} \frac{X_{k}(\omega)}{2^{k}}
$$

Поскольку случайные величины $\left\{X_{k}(\omega)\right\}_{k=1}^{\infty}$ принимают только лишь значения \pm 1 , это ряд со случайной расстановкой знаков и стохастические закономерности в расстановке знаков обусловлены свойствами набора $\left\{P_{n}\left(\delta_{1}, \ldots, \delta_{n}\right)\right\}$. Обозначим

$$
F_{S}(x)=\mathrm{P}(\omega: S(\omega) \leqslant x)
$$

функцию распределения суммы ряда (7). Это монотонно неубывающая неотрицательная функция; $F_{S}(x)=0$ при $x<-1$ и $F_{S}(x)=1$ при $x \geqslant 1$. Таким образом, отрезок $[-1,1]$ содержит спектр $F_{S}(x)$. 


\section{3. Результаты.}

Теорема 1. Функиия распределения $F_{S}(x)$ суммы ряда (7) непрерывна тогда $u$ только тогда, когда последовательность $\left\{P_{n}\left(t_{1}, \ldots, t_{n}\right)\right\}_{n=1}^{\infty}$ удовлетворяет условию

$$
\lim _{n \rightarrow \infty} P_{n}\left(t_{1}, \ldots, t_{n}\right)=0 \quad \forall \omega=\left(t_{1}, \ldots, t_{n}, \ldots\right) \in \Omega .
$$

Теорема 2 дает ответ на вопрос о том, насколько универсально представление непрерывных функций со спектром, содержащемся на отрезке $[-1,1]$ в виде функций распределения сумм рядов вида (7).

Теорема 2. Пусть $F(x), x \in[-1,1]$, - непрерывная монотонно неубывающая функиия и $F(-1)=0, F(1)=1$. Тогда на борелевском пространстве $(\Omega, \beta)$ мохно определить вероятность $\mathrm{P}$ таким образом, что функиия распределения $F_{S}(x)$ суммы $S$ ряда (7) есть $F(x)$.

Теоремы 3 и 4 представляют содержательную часть вероятностной процедуры, построенной в п. 2, по отношению к конструкции строго монотонных сингулярных функций, а именно, в этих теоремах приводятся условия на систему $\left\{P_{n}\left(t_{1}, \ldots, t_{n}\right)\right\}_{n=1}^{\infty}$, определяющую вероятность Р, чтобы функция распределения $F_{S}(x)$ суммы $S$ ряда $(7)$ была строго монотонной сингулярной функцией.

ТЕОрема 3. Функиия распределения $F_{S}(x)$ суммы ряда (7) строго монотонно возрастает на $[-1,1]$ тогда и только тогда, когда для $n=1,2, \ldots$

$$
P_{n}\left(t_{1}, \ldots, t_{n}\right)>0 \quad \forall t_{k}= \pm 1, \quad 1 \leqslant k \leqslant n .
$$

ТЕОрема 4. Пусть выполнено условие (8). Функция распределения $F_{S}(x)$ суммы ряда (7) сингулярна тогда и только тогда, когда для почти всех $x=t_{1} / 2+t_{2} / 2^{2}+\cdots$ $+t_{n} / 2^{n}+\cdots \in[-1,1]$

$$
\lim _{n \rightarrow \infty} 2^{n} P_{n}\left(t_{1}, \ldots, t_{n}\right)=0 .
$$

В качестве иллюстрации мы рассматриваем два стохастических способа расстановки знаков в ряде (7). Сначала вероятностная процедура рассматривается в ситуации, когда

$$
P_{n}\left(t_{1}, \ldots, t_{n}\right)=\prod_{k=1}^{n} p^{\left(1-t_{k}\right) / 2}(1-p)^{\left(1+t_{k}\right) / 2}, \quad 0<p<1, \quad p \neq \frac{1}{2} .
$$

Нетрудно показать, что последовательность $\left\{X_{k}(\omega)\right\}$, обеспечивающая стохастическую расстановку знаков в (7), является последовательностью равнораспределенных случайных величин с распределением

$$
\mathrm{P}\left(X_{n}(\omega)=\delta\right)=p^{(1-\delta) / 2}(1-p)^{(1+\delta) / 2}, \quad \delta= \pm 1, \quad n=1,2, \ldots
$$

Второй пример соответствует марковскому способу расстановки знаков в (7), а именно, пусть

$$
P_{1}\left(t_{1}\right)=\frac{1}{2}, \quad P_{n}\left(t_{1}, \ldots, t_{n}\right)=\frac{1}{2} \prod_{k=1}^{n-1} p\left(t_{k}, t_{k+1}\right), \quad n=2,3, \ldots,
$$

где $p\left(t_{k}, t_{k+1}\right)$ - элементы матрицы

$$
|| p\left(t_{k}, t_{k+1}\right) \mid=\left(\begin{array}{cc}
1-\alpha & \alpha \\
\alpha & 1-\alpha
\end{array}\right), \quad 0<\alpha<1, \quad \alpha \neq \frac{1}{2} .
$$

Подробно эти примеры рассмотрены в п. 5 . 


\section{4. Доказательства теорем.}

ДокаЗАТЕЛЬСТво ТЕОРЕМЫ 1 . Пусть $(8)$ имеет место, но $F_{S}(x)$ имеет точку разрьва $x_{0} \in[-1,1]$, т.е. для любого $\epsilon>0$ найдется $\Delta>0$ и

$$
F_{S}\left(x_{0}+\epsilon\right)-F_{S}\left(x_{0}-\epsilon\right) \geqslant \Delta>0 .
$$

Отсюда

$$
\mathrm{P}\left(\omega: x_{0}-\epsilon<S(\omega) \leqslant x_{0}+\epsilon\right) \geqslant \Delta>0 .
$$

По непрерьвности вероятности при $\epsilon \rightarrow 0$ имеем

$$
\mathrm{P}\left(\omega: S(\omega)=x_{0}\right) \geqslant \Delta>0 .
$$

В силу замечания к лемме 1 равенство $S(\omega)=x_{0}$ может реализоваться только одним $\omega_{0} \in \Omega$, поэтому

$$
\mathrm{P}\left(\omega: S(\omega)=x_{0}\right)=\mathrm{P}\left(\left\{\omega_{0}\right\}\right) \geqslant \Delta>0,
$$

что противоречит (8), которое, как это было показано ранее, означает отсутствие атомов в $(\Omega, \beta, \mathrm{P})$.

Обратно, пусть $F_{S}(x)$ непрерывна, т.е. для любого $x_{0} \in[-1,1]$

$$
F_{S}\left(x_{0}\right)-F_{S}(x) \rightarrow 0 \quad \text { при } x \rightarrow x_{0}-0 .
$$

Для $x \rightarrow x_{0}+0$ это выполняется всегда, так как функция распределения непрерывна справа. Далее имеем

$$
\mathrm{P}\left(\omega: x<S(\omega) \leqslant x_{0}\right) \rightarrow 0 \quad \text { при } x \rightarrow x_{0}-0 .
$$

По непрерьвности вероятности

$$
\mathrm{P}\left(\omega: S(\omega)=x_{0}\right)=0 \quad \forall x_{0} \in[-1,1] .
$$

В силу леммы 1 и замечания к ней отображение $S$ осушествляет биекцию $\Omega$ на $[-1,1]$; следовательно, из полученного равенства немедленно следует, что $\mathrm{P}(\{\omega\})=0$ для любого $\omega \in \Omega$, а это эквивалентно (8). Теорема установлена.

ДОКАЗАТЕЛЬСТВО ТЕОРЕМЫ 2. ПоЛОЖИм

$$
P_{n}\left(t_{1}, \ldots, t_{n}\right)=F\left(x_{n}+\frac{1}{2^{n}}\right)-F\left(x_{n}-\frac{1}{2^{n}}\right), \quad x_{n}=\frac{t_{1}}{2}+\cdots+\frac{t_{n}}{2^{n}} .
$$

Обрашаясь к лемме 2 , видим, что в правой части соотношения (13) стоит приращение функции $F(x)$ на образе $\left[x_{n}-1 / 2^{n}, x_{n}+1 / 2^{n}\right]$ цилиндрического множества $C_{n}\left(t_{1}, \ldots, t_{n}\right)$ при отображении $S$. Отметим также, что образами цилиндрических множеств $C_{n+1}\left(t_{1}\right.$, $\left.\ldots, t_{n},-1\right)$ и $C_{n+1}\left(t_{1}, \ldots, t_{n}, 1\right)$ будут, соответственно, отрезки $\left[x_{n}-1 / 2^{n}, x_{n}\right]$ и $\left[x_{n}\right.$, $\left.x_{n}+1 / 2^{n}\right]$. Действительно, например, для $S\left(C_{n+1}\left(t_{1}, \ldots, t_{n},-1\right)\right)$ из леммы 2 имеем

$$
S\left(C_{n+1}\left(t_{1}, \ldots, t_{n},-1\right)\right)=\left\{x \in[-1,1]: x_{n+1}-\frac{1}{2^{n+1}} \leqslant x \leqslant x_{n+1}+\frac{1}{2^{n+1}}\right\},
$$

при этом

$$
x_{n+1}=\frac{t_{1}}{2}+\cdots+\frac{t_{n}}{2^{n}}-\frac{1}{2^{n+1}}=x_{n}-\frac{1}{2^{n+1}}
$$


и, следовательно,

$$
S\left(C_{n+1}\left(t_{1}, \ldots, t_{n},-1\right)\right)=\left\{x \in[-1,1]: x_{n}-\frac{1}{2^{n}} \leqslant x \leqslant x_{n}\right\} .
$$

В силу этого, условие согласованности набора конечномерных вероятностных распределений, задаваемых (13),

$$
P_{n}\left(t_{1}, \ldots, t_{n}\right)=P_{n+1}\left(t_{1}, \ldots, t_{n},-1\right)+P_{n+1}\left(t_{1}, \ldots, t_{n},+1\right)
$$

есть следствие того, что $F(x)$ непрерьвна и прирашение $F(x)$ на отрезке $\left[x_{n}-1 / 2^{n}\right.$, $\left.x_{n}+1 / 2^{n}\right]$ есть сумма приращений этой функции на отрезках $\left[x_{n}-1 / 2^{n}, x_{n}\right]$ и $\left[x_{n}\right.$, $\left.x_{n}+1 / 2^{n}\right]$.

Покажем сейчас, что приращения $F(x)$ и $F_{S}(x)$ равны на любом отрезке $\left[x_{n}-1 / 2^{n}\right.$, $x_{n}+1 / 2^{n}$ ] для любого $n=1,2, \ldots$ Действительно,

$$
\begin{aligned}
\left.\Delta F_{S}(x)\right|_{\left[x_{n}-\frac{1}{2^{n}}, x_{n}+\frac{1}{\left.2^{n}\right]}\right.} & =F_{S}\left(x_{n}+\frac{1}{2^{n}}\right)-F_{S}\left(x_{n}-\frac{1}{2^{n}}\right) \\
& =\mathrm{P}\left(\omega: x_{n}-\frac{1}{2^{n}}<S(\omega) \leqslant x_{n}+\frac{1}{2^{n}}\right)=\mathrm{P}\left(C_{n}\left(t_{1}, \ldots, t_{n}\right)\right) \\
& =P_{n}\left(t_{1}, \ldots, t_{n}\right)=\left.\Delta F(x)\right|_{\left[x_{n}-\frac{1}{2^{n}}, x_{n}+\frac{1}{2^{n}}\right]} .
\end{aligned}
$$

Учитьвая теперь, что $F_{S}(-1)=F(-1)=0$, приходим к вьводу, что $F_{S}(x)=F(x)$ при $x=x_{n}+1 / 2^{n}, n=1,2, \ldots$. Заметим теперь, что

$$
x=x_{n}+\frac{1}{2^{n}}=\frac{m}{2^{n-1}}, \quad-2^{n-1}+1 \leqslant m \leqslant 2^{n-1}-1, \quad \text { где } m \text { нечетны. }
$$

Но числа вида $\left\{m / 2^{n-1}\right\}_{n=1}^{\infty}$ распределены всюду плотно на $[-1,1]$. Следовательно, учитьвая непрерьвность $F(x)$ и, как следствие, непрерывность $F_{S}(x)((13)$ и теорема 1), убеждаемся в равенстве $F(x)=F_{S}(x)$. Теорема установлена.

ДоКАЗАТЕЛЬСТво ТЕОРЕмЫ 3. Пусть (9) вьполнено, но нашлись $x_{1}, x_{2} \in[-1,1]$, $x_{1}<x_{2}$ такие, что $F_{S}\left(x_{1}\right)=F_{S}\left(x_{2}\right)$. Это влечет $\mathrm{P}\left(\omega: x_{1}<S(\omega) \leqslant x_{2}\right)=0$. Обозначим $\bar{x}=\left(x_{1}+x_{2}\right) / 2$ и $\epsilon=\left(x_{2}-x_{1}\right) / 2$. В силу леммы 1 найдется $\bar{\omega}=\left(\bar{t}_{1}, \ldots, \bar{t}_{k}, \ldots\right)$ такое, что

$$
\bar{x}=S(\bar{\omega})=\sum_{k=1}^{\infty} \frac{\bar{t}_{k}}{2^{k}}
$$

Подберем $n=n(\epsilon)$ так, чтобы

$$
\left|S(\bar{\omega})-\sum_{k=1}^{n} \frac{\bar{t}_{k}}{2^{k}}\right| \leqslant \sum_{k=n+1} \frac{1}{2^{k}}=\frac{\epsilon}{2}
$$

и рассмотрим цилиндр $C_{n}\left(\bar{t}_{1}, \ldots, \bar{t}_{n}\right)$. Для произвольного $\omega \in C_{n}\left(\bar{t}_{1}, \ldots, \bar{t}_{n}\right), \omega=\left(\bar{t}_{1}\right.$, $\left.\ldots, \bar{t}_{n}, t_{n+1}, \ldots\right), t_{k}= \pm 1, k \geqslant n+1$, имеем

$$
|S(\omega)-\bar{x}|=|S(\omega)-S(\bar{\omega})|=\left|\sum_{k=n+1}^{\infty} \frac{t_{k}-\bar{t}_{k}}{2^{k}}\right| \leqslant \epsilon .
$$


Поэтому

$$
C_{n}\left(\bar{t}_{1}, \ldots, \bar{t}_{n}\right) \subset A=\{\omega:|S(\omega)-\bar{x}| \leqslant \epsilon\}=\left\{\omega: x_{1} \leqslant S(\omega) \leqslant x_{2}\right\}
$$

И

$$
\mathrm{P}\left(C_{n}\left(\bar{t}_{1}, \ldots, \bar{t}_{n}\right)\right)=P_{n}\left(\bar{t}_{1}, \ldots, \bar{t}_{n}\right) \leqslant \mathrm{P}(A) .
$$

С одной стороны, $\mathrm{P}(A)=0$, с другой же стороны, в силу (9) $P_{n}\left(\bar{t}_{1}, \ldots, \bar{t}_{n}\right)>0$. Итак, (9) достаточно для строго монотонного возрастания $F_{S}(x)$.

Обратно, пусть (9) не выполняется; тогда найдется набор $\left(\delta_{1}, \ldots, \delta_{n}\right)$ такой, что

$$
P_{n}\left(\delta_{1}, \ldots, \delta_{n}\right)=0 .
$$

Рассмотрим произвольные $x_{1}, x_{2} \in\left(x_{n}-1 / 2^{n}, x_{n}+1 / 2^{n}\right)$, где $x_{n}=\delta_{1} / 2+\cdots+\delta_{n} / n$, и покажем, что $F_{S}\left(x_{1}\right)=F_{S}\left(x_{2}\right)$. Действительно,

$$
\begin{aligned}
F_{S}\left(x_{2}\right)-F_{S}\left(x_{1}\right) & =\mathrm{P}\left(\omega: x_{1}<S(\omega) \leqslant x_{2}\right) \leqslant \mathrm{P}\left(\omega: x_{n}-\frac{1}{2^{n}}<S(\omega) \leqslant x_{n}+\frac{1}{2^{n}}\right) \\
& =\mathrm{P}\left(C_{n}\left(\delta_{1}, \ldots, \delta_{n}\right)\right)=P_{n}\left(\delta_{1}, \ldots, \delta_{n}\right)=0 .
\end{aligned}
$$

Следовательно, строго монотонное возрастание функции $F_{S}(x)$ нарушается. Теорема доказана.

ДОКАЗАТЕЛЬСТво ТЕОРЕМЫ 4 . Пусть $F_{S}(x)$ - сингулярна; тогда $F_{S}^{\prime}(x)=0$ почти всюду на отрезке $[-1,1]$, т.е. найдется множество $U \subset[-1,1]$ полной меры такое, что $F_{S}^{\prime}(x)=0$ для всех $x \in U$. Заметим, что можно пренебречь двоично-рациональными точками в $U$, т.е. точками вида $x=t_{1} / 2+t_{k} / 2^{k}, t_{k}= \pm 1$, так как они образуют множества нулевой лебеговой меры.

Для произвольной точки $x=t_{1}^{(x)} / 2+t_{k}^{(x)} / 2^{k}+\cdots \in U$ построим систему стягиваюшихся отрезков вида

$$
\begin{gathered}
\left\{\left[x_{1}^{(n)}, x_{2}^{(n)}\right]: x_{1}^{(n)}=\frac{t_{1}^{(x)}}{2}+\cdots+\frac{t_{n}^{(x)}}{2^{n}}-\frac{1}{2^{n+1}}-\cdots,\right. \\
\left.x_{2}^{(n)}=\frac{t_{1}^{(x)}}{2}+\cdots+\frac{t_{n}^{(x)}}{2^{n}}+\frac{1}{2^{n+1}}+\cdots\right\}_{n=1}^{\infty} .
\end{gathered}
$$

Длина отрезков $\left[x_{1}^{(n)}, x_{2}^{(n)}\right] x_{2}^{(n)}-x_{1}^{(n)}=1 / 2^{n-1}$ и

$$
x=\bigcap_{n=1}^{\infty}\left[x_{1}^{(n)}, x_{2}^{(n)}\right] .
$$

Так как в точке $x$ производная $F_{S}^{\prime}(x)$ сушествует и $F_{S}^{\prime}(x)=0$, получаем

$$
0=F_{S}^{\prime}(x)=\lim _{n \rightarrow \infty} \frac{F_{S}\left(x_{2}^{(n)}\right)-F_{S}\left(x_{1}^{(n)}\right)}{x_{2}^{(n)}-x_{1}^{(n)}}=\lim _{n \rightarrow \infty} 2^{n} \mathrm{P}\left(\omega: x_{1}^{(n)}<S(\omega) \leqslant x_{2}^{(n)}\right) .
$$

Из леммы 2 , используя замечание к лемме 1 , можно сделать вьвод, что прообразом отрезка $\left[x_{1}^{(n)}, x_{2}^{(n)}\right]$ является цилиндрическое множество $C_{n}\left(t_{1}^{(x)}, \ldots, t_{n}^{(x)}\right)$, поэтому

$$
\mathrm{P}\left(\omega: x_{1}^{(n)}<S(\omega) \leqslant x_{2}^{(n)}\right)=\mathrm{P}\left(C_{n}\left(t_{1}^{(x)}, \ldots, t_{n}^{(x)}\right)\right)=P_{n}\left(t_{1}^{(x)}, \ldots, t_{n}^{(x)}\right),
$$


а значит, для почти всех $x \in[-1,1]$

$$
\lim _{n \rightarrow \infty} 2^{n} P_{n}\left(t_{1}^{(x)}, \ldots, t_{n}^{(x)}\right)=0 .
$$

Необходимость условия (10) сингулярности $F_{S}(x)$ установлена.

Докажем теперь достаточность. Пусть условие $(10)$ вьполнено. Так как $F_{S}(x)-$ функция распределения ряда, она монотонно возрастает, а следовательно, по теореме Лебега [4] ее производная существует и конечна почти всюду на $[-1,1]$. Для произвольной точки $x=t_{1}^{(x)} / 2+\cdots+t_{k}^{(x)} / 2^{k}+\cdots$, для которой существует конечная производная, построим набор стягивающихся отрезков $\left\{\left[x_{1}^{(n)}, x_{2}^{(n)}\right]\right\}_{n=1}^{\infty}$ так же, как было описано выше. Тогда имеем

$$
F_{S}^{\prime}(x)=\lim _{n \rightarrow \infty} \frac{F_{S}\left(x_{2}^{(n)}\right)-F_{S}\left(x_{1}^{(n)}\right)}{x_{2}^{(n)}-x_{1}^{(n)}}=\lim _{n \rightarrow \infty} 2^{n} P_{n}\left(t_{1}^{(x)}, \ldots, t_{n}^{(x)}\right),
$$

а в силу условия (10) для почти всех $x \in[-1,1], x=t_{1} / 2+\cdots+t_{n} / 2^{n}+\cdots$,

$$
\lim _{n \rightarrow \infty} 2^{n} P_{n}\left(t_{1}, \ldots, t_{n}\right)=0
$$

Поскольку пересечение двух множеств полной меры есть множество полной меры, то $F_{S}^{\prime}(x)=0$ почти всюду, а условие $(8)$ обеспечивает непрерьвность $F_{S}(x)$, что и обеспечивает сингулярность $F_{S}(x)$. Теорема доказана.

5. Примеры. Сейчас мы обратимся к двум частным способам стохастической расстановки знаков.

ПримеР 1 (функция Салема). Пусть набор $\left\{P_{n}\left(t_{1}, \ldots, t_{n}\right)\right\}$ задается посредством (11). Нетрудно видеть, что условия $(8),(9)$ вьполняются, так что $F_{S}(x)$ - непрерывная строго монотонно возрастающая на $[-1,1]$ функция. Обрашаясь теперь к теореме 4 , покажем, что $F_{S}(x)$ сингулярная функция при $p \neq 1 / 2$. Действительно, имея в виду условие $(10)$, пусть $x=t_{1} / 2+\cdots+t_{n} / 2^{n}+\cdots \in[-1,1]$ и $n_{x}-$ количество $(-1)$ в наборе $\left(t_{1}, \ldots, t_{n}\right)$. Имеем согласно $(11)$

$$
2^{n} P_{n}\left(t_{1}, \ldots, t_{n}\right)=2^{n} p^{n_{x}}(1-p)^{n-n_{x}} .
$$

Согласно известной теоремы о "нормальных" числах (см., например, [8, с. 39])

$$
\lim _{n \rightarrow \infty} \frac{n_{x}}{n}=\frac{1}{2}
$$

для почти всех $x \in[-1,1]$, так что для всех $\epsilon>0$ и $n>N(\epsilon ; x)$

$$
\frac{1}{2}-\epsilon<\frac{n_{x}}{n}<\frac{1}{2}+\epsilon
$$

Поэтому

$$
\begin{aligned}
2^{n} P_{n}\left(t_{1}, \ldots, t_{n}\right) & =(2(1-p))^{n}\left(\left(\frac{p}{(1-p)}\right)^{n_{x} / n}\right)^{n} \\
& \leqslant\left(2(1-p)\left(\frac{p}{1-p}\right)^{1 / 2 \pm \epsilon}\right)^{n}=\left((4(1-p) p)^{1 / 2}\left(\frac{p}{1-p}\right)^{ \pm \epsilon}\right)^{n} .
\end{aligned}
$$


Здесь “+” или “-” выбираются в соответствии с $p>1 / 2$ или $p<1 / 2$. Поскольку $[4 p(1-p)]^{\frac{1}{2}}<1$, при достаточно малом $\epsilon>0$ и всех $n>N(\epsilon ; x)$ получаем

$$
(4(1-p) p)^{1 / 2}\left(\frac{p}{1-p}\right)^{ \pm \epsilon}<1
$$

что и обеспечивает вьполнение (11) для почти всех $x \in[-1,1]$ при $p \neq 1 / 2$.

Следующая наша цель - это показать, что $F_{S}(x)$ является функцией Салема [2]. Важная информация содержится в следующей лемме.

Лемма 3. Пусть выполнено (11). Тогда

1) для функиии распределения $F_{S}(x)$ ряда (7) имеем соотношение

$$
F_{S}(x)=p F_{S}(2 x+1)+(1-p) F_{S}(2 x-1) ;
$$

2) для преобразования Фурье-Стилтьеса $\widehat{F}_{S}(z)$ функиии распределения $F_{S}(x)$ имеем для $z \in \mathbb{C}$

$$
\widehat{F}_{S}(z)=\prod_{n=1}^{\infty} Q\left(\frac{z}{2^{n}}\right), \quad Q(u)=(1-p) e^{i u}+p e^{-i u}
$$

ДокАЗАТЕЛЬСТво. 1) При выполнении (11) система координатных случайных величин $\left\{X_{k}(\omega)\right\}_{k=1}^{\infty}$ является системой независимых одинаково распределенньх случайных величин. Поэтому

$$
\begin{aligned}
F_{S}(x) & =\mathrm{P}(\omega: S(\omega) \leqslant x) \\
& =\mathrm{P}\left(X_{1}=-1 ;-\frac{1}{2}+\frac{X_{2}}{2^{2}}+\cdots \leqslant x\right)+\mathrm{P}\left(X_{1}=+1 ; \frac{1}{2}+\frac{X_{2}}{2^{2}}+\cdots \leqslant x\right) \\
& =p F_{S}(2 x+1)+(1-p) F_{S}(2 x-1) .
\end{aligned}
$$

2) Имеем на основании (15)

$$
\begin{aligned}
\widehat{F}_{S}(z) & =\int_{-\infty}^{\infty} e^{i z x} d F_{S}(x)=p \int_{-\infty}^{\infty} e^{i z x} d F_{S}(2 x+1)+(1-p) \int_{-\infty}^{\infty} e^{i z x} d F_{S}(2 x-1) \\
& =p e^{-i \frac{z}{2}} \int_{-\infty}^{\infty} e^{i \frac{z}{2} t} d F_{S}(t)+(1-p) e^{i \frac{z}{2}} \int_{-\infty}^{\infty} e^{i \frac{z}{2} t} d F_{S}(t) \\
& =Q\left(\frac{z}{2}\right) \widehat{F}_{S}\left(\frac{z}{2}\right)
\end{aligned}
$$

Итерируя полученное соотношение, имеем

$$
\widehat{F}_{S}(z)=\widehat{F}_{S}\left(\frac{z}{2^{n}}\right) \prod_{k=1}^{n} Q\left(\frac{z}{2^{n}}\right)
$$

При $n \rightarrow \infty \widehat{F}_{S}\left(z / 2^{n}\right) \rightarrow 1 ; \prod_{k=1}^{n} Q\left(z / 2^{n}\right) \rightarrow \prod_{n=1}^{\infty} Q\left(z / 2^{n}\right)$ равномерно на любом компакте в $\mathbb{C}[9$, с. 277$]$. 
ЗАМЕчАНИЕ 1. При $p=1 / 2$, как видно из $(16), \widehat{F}_{S}(z)=\sin z / z$ и, следовательно, $F_{S}(x)=x / 2+1 / 2$, т.е. функция распределения равномерного вероятностного распределения на $[-1,1]$. В этом случае $P_{n}\left(t_{1}, \ldots, t_{n}\right)=1 / 2^{n}, n=1,2, \ldots$, т.е. знаки в $(7)$ расставляются независимо и равновероятно.

ЗАмечаниЕ 2. Сравнивая выражение $(16)$ для $\widehat{F}_{S}(z)$ при $p \neq 1 / 2$ с формулой для преобразования Фурье-Стилтьеса функции Салема [2], адаптированной к отрезку $[-1,1]$ (в [2] получены только формулы для коэффициентов Фурье-Стилтьеса), убеждаемся в их совпадении. Привлекая теперь теорему единственности для преобразований $Ф у-$ рье-Стилтьеса [8], приходим к выводу, что функция распределения суммы ряда (7) в данном частном случае $(11), p \neq 1 / 2$, есть в точности функция Салема, конструктивная процедура построения которой приведена в [2] (см. также [4, с. 59]).

Пример 2. Пусть набор $\left\{P_{n}\left(t_{1}, \ldots, t_{n}\right)\right\}$ задается посредством (12). В этом случае координатные случайные величины $\left\{X_{k}\right\}_{k=1}^{\infty}$ образуют марковскую цепь с начальньп распределением $(1 / 2,1 / 2)$ и матрицей переходных вероятностей $\left(\begin{array}{cc}1-\alpha & \alpha \\ \alpha & 1-\alpha\end{array}\right)$. В [10] получена удобная формула для $P_{n}\left(t_{1}, \ldots, t_{n}\right):$ для $n=2,3, \ldots$

$$
P_{n}\left(t_{1}, \ldots, t_{n}\right)=\frac{1}{2^{n} \operatorname{ch}^{n-1} J} e^{J \sum_{k=1}^{n-1} t_{k} t_{k+1}}, \quad J=\frac{1}{2} \ln \frac{1-\alpha}{\alpha} .
$$

Как нетрудно видеть из (17), условия (8) и (9) теорем 1 и 3 вьполняются; следовательно, $F_{S}(x)$ является непрерьвной строго монотонно возрастающей функцией. Покажем, что при $\alpha \neq 1 / 2 F_{S}(x)$ является сингулярной функцией.

Левая часть соотношения (10) теоремы 4 с учетом (17) может быть записана в виде

$$
\frac{\operatorname{ch} J}{e^{J t_{1}}} \lim _{n \rightarrow \infty} \exp \left\{J\left(t_{1}+\sum_{k=1}^{n-1} t_{k} t_{k+1}\right)-n \ln \operatorname{ch} J\right\} .
$$

Остается показать, что для почти всех $x=t_{1} / 2+\cdots+t_{n} / 2^{n}+\cdots \in[-1,1]$ предел в $(18)$ равен нулю.

Рассмотрим отображение $A:[-1,1] \rightarrow[-1,1]$, определяемое следуюшим образом: пусть $x=t_{1} / 2+\cdots+t_{n} / 2^{n}+\cdots ;$ тогда

$$
u=A(x), \quad \text { где } \quad u=\frac{t_{1}}{2}+\frac{t_{1} t_{2}}{2^{2}}+\cdots+\frac{t_{n-1} t_{n}}{2^{n}}+\cdots \in[-1,1] .
$$

Обозначим $s_{1}=t_{1}, s_{2}=t_{1} t_{2}, \ldots, s_{n}=t_{n-1} t_{n}, \ldots-$ коэффициенты бинарного разложения $u$. Тогда в новых обозначениях предел в (18) запишется в виде

$$
\lim _{n \rightarrow \infty} \exp \left\{J \sum_{k=1}^{n} s_{k}-n \ln \operatorname{ch} J\right\} .
$$

Нетрудно показать, что для почти всех $u \in[-1,1], u=s_{1} / 2+s_{2} / 2^{2}+\cdots+s_{n} / 2^{n}+\cdots$, этот предел равен нулю. Действительно, обозначим $n_{u}-$ количество $(-1)$ в наборе $\left(s_{1}, \ldots, s_{n}\right)$, так что $\left(n-n_{u}\right)$ - количество $(+1)$ в этом наборе. В таком случае рассматриваемьй предел запишется в виде

$$
\lim _{n \rightarrow \infty} \exp \left\{n\left[2 J\left(\frac{1}{2}-\frac{n_{u}}{n}\right)-\ln \operatorname{ch} J\right]\right\} .
$$


С учетом того, что $\ln \operatorname{ch} J>0$ для $J \neq 0(\alpha \neq 1 / 2)$ и теоремы о "нормальных" числах $[8$, c. 39] для всех достаточно больших $n$ и почти всех $u \in[-1,1]$

$$
2 J\left(\frac{1}{2}-\frac{n_{u}}{n}\right)-\ln \operatorname{ch} J<0
$$

что и приводит к желаемому результату.

Дальнейшая наша цель - показать, что тот же вывод справедлив и для почти всех $x=t_{1} / 2+\cdots+t_{n} / 2^{n}+\cdots \in[-1,1]$. Обратимся к отображению $A$.

Лемма 5. Отображсение $A:[-1,1] \rightarrow[-1,1]$, определяемое формулой (19) сюрдективно и является биекцией вне множсества рациональных чисел.

ДокАЗАТЕЛЬСТВо. Пусть $u=s_{1} / 2+\cdots+s_{n} / 2^{n}+\cdots \in[-1,1], s_{n}= \pm 1$, произвольное. Рассмотрим $x=s_{1} / 2+s_{1} s_{2} / 2^{2}+\cdots+s_{1} s_{2} \ldots s_{n} / 2^{n}+\cdots \in[-1,1]$. Имеем

$$
\begin{aligned}
A(x) & =\frac{s_{1}}{2}+\frac{s_{1}^{2} s_{2}}{2^{2}}+\frac{\left(s_{1} s_{2}\right)^{2} s_{3}}{2^{3}}+\cdots+\frac{\left(s_{1} s_{2} \ldots s_{n-1}\right)^{2} s_{n}}{2^{n}}+\cdots \\
& =\frac{s_{1}}{2}+\frac{s_{2}}{2^{2}}+\cdots+\frac{s_{n}}{2^{n}}+\cdots=u
\end{aligned}
$$

и сюръективность установлена.

Покажем теперь, что "слипание" образов отображения $A$ происходит на рациональных $x \in[-1,1]$ и образом в этом случае является также рациональное $u \in[-1,1]$. Итак, пусть

$$
\begin{gathered}
A\left(x_{1}\right)=A\left(x_{2}\right), \quad x_{1}=\frac{t_{1}^{(1)}}{2}+\cdots+\frac{t_{k}^{(1)}}{2^{k}}+\cdots, \quad x_{2}=\frac{t_{1}^{(2)}}{2}+\cdots+\frac{t_{k}^{(2)}}{2^{k}}+\cdots \quad \text { и } \\
n=\min \left(k: t_{k}^{(1)} \neq t_{k}^{(2)}\right), \quad \text { т.е. } t_{1}^{(1)}=t_{1}^{(2)}, \ldots, \quad t_{n-1}^{(1)}=t_{n-1}^{(2)}, \quad t_{n}^{(1)} \neq t_{n}^{(2)} .
\end{gathered}
$$

Тогда равенство $A\left(x_{1}\right)=A\left(x_{2}\right)$ с учетом (19) влечет

$$
\frac{t_{n-1}^{(1)} t_{n}^{(1)}}{2^{n}}+\frac{t_{n}^{(1)} t_{n+1}^{(1)}}{2^{n+1}}+\cdots=\frac{t_{n-1}^{(2)} t_{n}^{(2)}}{2^{n}}+\frac{t_{n}^{(2)} t_{n+1}^{(2)}}{2^{n+1}}+\cdots
$$

Примем для определенности $t_{n-1}^{(1)}=t_{n-1}^{(2)}=1, t_{n}^{(1)}=1, t_{n}^{(2)}=-1$. Имеем из $(20)$

$$
\left(t_{n}^{(1)}-t_{n}^{(2)}\right)+\frac{t_{n}^{(1)} t_{n+1}^{(1)}-t_{n}^{(2)} t_{n+1}^{(2)}}{2}+\frac{t_{n+1}^{(1)} t_{n+2}^{(1)}-t_{n+1}^{(2)} t_{n+2}^{(2)}}{2^{2}}+\cdots=0
$$

или

$$
2+\frac{t_{n+1}^{(1)}+t_{n+1}^{(2)}}{2}+\frac{t_{n+1}^{(1)} t_{n+2}^{(1)}-t_{n+1}^{(2)} t_{n+2}^{(2)}}{2^{2}}+\cdots=0 .
$$

Отсюда с необходимостью получим

$$
\left\{\begin{array} { l } 
{ t _ { n + 1 } ^ { ( 1 ) } = - 1 , } \\
{ t _ { n + 1 } ^ { ( 2 ) } = - 1 , }
\end{array} \quad \left\{\begin{array} { l } 
{ t _ { n + 2 } ^ { ( 1 ) } = 1 , } \\
{ t _ { n + 2 } ^ { ( 2 ) } = - 1 , }
\end{array} \quad \cdots \quad \left\{\begin{array}{l}
t_{n+k}^{(1)}=(-1)^{k} \\
t_{n+k}^{(2)}=-1
\end{array}\right.\right.\right.
$$


Поэтому, обозначая $t_{1}^{(1)}=t_{1}^{(2)}=t_{1}, \ldots, t_{n-1}^{(1)}=t_{n-1}^{(2)}=t_{n-1}$, имеем

$$
\begin{aligned}
& x_{1}=\frac{t_{1}}{2}+\frac{t_{2}}{2^{2}}+\cdots+\frac{t_{n-2}}{2^{n-2}}+\frac{1}{2^{n-1}}+\frac{1}{2^{n}}-\frac{1}{2^{n+1}}+\cdots+\frac{(-1)^{k}}{2^{n+k}}+\cdots, \\
& x_{2}=\frac{t_{1}}{2}+\frac{t_{2}}{2^{2}}+\cdots+\frac{t_{n-2}}{2^{n-2}}+\frac{1}{2^{n-1}}-\frac{1}{2^{n}}-\frac{1}{2^{n+1}}-\cdots-\frac{1}{2^{n+k}}+\cdots .
\end{aligned}
$$

Очевидно, обе точки рациональные, равно как и их образ

$$
\begin{aligned}
u=A\left(x_{1}\right)=A\left(x_{2}\right) & =\frac{t_{1}}{2}+\frac{t_{1} t_{2}}{2^{2}}+\cdots+\frac{t_{n-3} t_{n-2}}{2^{n-2}}+\frac{t_{n-2}}{2^{n-1}}-\frac{1}{2^{n}}+\frac{1}{2^{n+1}}+\cdots \\
& =\frac{t_{1}}{2}+\frac{t_{1} t_{2}}{2^{2}}+\cdots+\frac{t_{n-3} t_{n-2}}{2^{n-2}}+\frac{t_{n-2}}{2^{n-1}} .
\end{aligned}
$$

Таким образом, отображение $A$ не является инъективным и нарушение инъективности происходит на множестве рациональных точек отрезка $[-1,1]$. Исключив рациональные точки, мы имеем на оставшейся части отрезка $[-1,1]$, которая имеет полную лебегову меру, биективность отображения $A$. Лемма установлена.

На множестве иррациональных точек отрезка $[-1,1]$ определим обратное отображение $A^{-1}(u)$ : пусть $u=s_{1} / 2+\cdots+s_{n} / 2^{n}+\cdots$; тогда

$$
x=A^{-1}(u)=\frac{s_{1}}{2}+\frac{s_{1} s_{2}}{2^{2}}+\cdots+\frac{s_{1} s_{2} \cdots s_{n}}{2^{n}}+\cdots .
$$

Лемма 6. Отображсение $A^{-1}$ сохраняет меру Лебега.

ДокаЗАТЕЛЬСтво. Рассмотрим подмножество $M_{n}\left(\delta_{1}, \ldots, \delta_{n}\right) \subset[-1,1]$ вида

$$
\begin{gathered}
M_{n}\left(\delta_{1}, \ldots, \delta_{n}\right)=\left\{u \in[-1,1]: u=\frac{\delta_{1}}{2}+\cdots+\frac{\delta_{n}}{2^{n}}+\frac{s_{n+1}}{2^{n+1}}+\cdots,\right. \\
\delta_{k}= \pm 1,1 \leqslant k \leqslant n, \text { фиксированы, } \\
\left.s_{k}= \pm 1, k \geqslant n+1, \text { произвольные }\right\} \\
=\left\{u \in[-1,1]: u=\frac{m}{2^{n}}+\frac{1}{2^{n}}\left(\frac{s_{n+1}}{2}+\frac{s_{n+2}}{2^{2}}+\cdots\right),\right. \\
\left.m=\delta_{1} 2^{n-1}+\cdots+\delta_{n}\right\},
\end{gathered}
$$

т.е. $M_{n}\left(\delta_{1}, \ldots, \delta_{n}\right)$ есть отрезок вида $\left[m / 2^{n}-1 / 2^{n}, m / 2^{n}+1 / 2^{n}\right]$, так что лебегова мера $\lambda\left(M_{n}\left(\delta_{1}, \ldots, \delta_{n}\right)\right)=1 / 2^{n-1}$.

Далее имеем согласно $(21)$ на множестве иррациональных точек $M_{n}\left(\delta_{1}, \ldots, \delta_{n}\right)$

$$
\begin{aligned}
A^{-1}\left(M_{n}\left(\delta_{1}, \ldots, \delta_{n}\right)\right)= & \left\{x \in[-1,1]: x=\frac{\delta_{1}}{2}+\frac{\delta_{1} \delta_{2}}{2^{2}}+\cdots+\frac{\delta_{1} \delta_{2} \cdots \delta_{n}}{2^{n}}\right. \\
& \left.+\frac{\delta_{1} \delta_{2} \cdots \delta_{n} s_{n+1}}{2^{n+1}}+\cdots\right\} \\
= & \left\{x \in[-1,1]: x=\frac{l}{2^{n}}+\frac{\delta_{1} \delta_{2} \cdots \delta_{n}}{2^{n}}\left(\frac{s_{n+1}}{2}+\frac{s_{n+1} s_{n+2}}{2^{2}}+\cdots\right),\right. \\
& \left.l=\delta_{1} 2^{n-1}+\cdots+\delta_{1} \delta_{2} \cdots \delta_{n}\right\} \\
= & \left\{x \in[-1,1]: x=\frac{l}{2^{n}}+\frac{1}{2^{n}} A^{-1}([-1,1])\right\} .
\end{aligned}
$$


Отсюда и из свойств биективности отображения $A$ на множестве иррациональных точек отрезка $[-1,1]$ заключаем, что

$$
\lambda\left(A^{-1}\left(M_{n}\left(\delta_{1}, \ldots, \delta_{n}\right)\right)\right)=\lambda\left\{x \in[-1,1]:\left|x-\frac{l}{2^{n}}\right| \leqslant \frac{1}{2^{n}}\right\}=\frac{1}{2^{n-1}} .
$$

Таким образом, на отрезках вида $\left[m / 2^{n}-1 / 2^{n}, m / 2^{n}+1 / 2^{n}\right]$ для всех $n=1,2, \ldots$ отображение $A^{-1}$ сохраняет меру Лебега. Поскольку точки вида $m / 2^{n}, m \in \mathbb{Z}, n \in \mathbb{N}$, всюду плотно расположены на $[-1,1]$, а отображение $A^{-1}$ биективно вне множества рациональных точек, которое имеет меру нуль, отсюда следует, что отображение $A^{-1}$ сохраняет меру Лебега. Лемма установлена.

СлЕДСТВИЕ. Предел (18) равен нулю для почти всех $x \in[-1,1], x=t_{1} / 2+\cdots$ $+t_{n} / 2^{n}+\cdots$.

ДоКАЗАТЕЛЬСТво. Действительно, нами установлено, что (18) равен нулю для почти всех $u=A(x) \in[-1,1]$. Но согласно лемме 6 отображение $x=A^{-1}(u)$ переводит множество полной меры в множество полной меры; следовательно, равенство нулю для почти всех $u \in[-1,1]$ соответствует почти всем $x=t_{1} / 2+\cdots+t_{n} / 2^{n}+\cdots \in[-1,1]$.

\section{СПИСОК ЦИТИРОВАННОЙ ЛИТЕРАТУРЫ}

[1] Minkowski H. Zur Geometrie der Zahlen // Gesammelte Abhandlungen. 1911. V. 2. P. 50-51.

[2] Salem R. On some singular monotonic functions which are strictly increasing // Trans. Amer. Math. Soc. 1943. V. 53. P. 427-439.

[3] Denjoy A. Sur une fonction réele de Minkowski // J. Math. Pures Appl. 1938. V. 17. P. 105-151.

[4] Рисс Ф., Секефальви-Надь Б. Лекции по функциональному анализу. 2-е изд. М.: Мир, 1979.

[5] de Rham G. Sur quelques courbes definies par des equations fonctionelles // Rend. Sem. Matem. Univ. Torino. 1956-1957. № 16. P. 101-113.

[6] Сакс С. Теория интеграла. М.: ИЛ, 1949.

[7] Партасарати К. Введение в теорию вероятностей и теорию меры. М.: Мир, 1983.

[8] Ламперти Дж. Вероятность. М.: Наука, 1973.

[9] Маркушевич А. И. Теория аналитических функций. Т. 1. М.: Наука, 1967.

[10] Рябинин А. А. О характеристических функциях вероятностных распределений сумм со случайной расстановкой знаков // Теория вероятн. и ее примен. 2000. Т. 45. № 4. С. 773-776. 